\title{
Volumes of highly twisted knots and links
}

\author{
JESSICA S PURCELL
}

\begin{abstract}
We show that for a large class of knots and links with complements in $S^{3}$ admitting a hyperbolic structure, we can determine bounds on the volume of the link complement from combinatorial information given by a link diagram. Specifically, there is a universal constant $\mathrm{C}$ such that if a knot or link admits a prime, twist reduced diagram with at least 2 twist regions and at least $C$ crossings per twist region, then the link complement is hyperbolic with volume bounded below by 3.3515 times the number of twist regions in the diagram. $\mathrm{C}$ is at most 113.
\end{abstract}

57M25, 57M50

\section{Introduction}

Given a diagram of a knot or link, our goal is to determine geometric information about the complement of that link in $S^{3}$. In particular, if the complement admits a hyperbolic structure, then by Mostow-Prasad rigidity that structure is unique. We ought to be able to make explicit statements about the geometry of this link complement, including statements about its volume. However, such results based purely on a diagram seem to be rare.

Given a particular diagram, there are examples of volume computations. For example, Cao and Meyerhoff proved the smallest volume knot or link complement was the figure eight knot complement [4]. Given a simple diagram, computer software such as Weeks' SnapPea can often compute a hyperbolic structure, including the volume [16].

For particular classes of knots and links, other results on volume have been determined. Lackenby proved that in the special case in which a knot or link is alternating, the volume of the complement is bounded above and below by the twist number of a diagram [10]. In fact, the upper bound is valid for all knots, not just alternating. This upper bound was further improved by Agol and D Thurston in an appendix to Lackenby's paper. Additionally, they found a sequence of links with volume approaching the upper bound.

Recently, the lower bound has been improved by work of Agol, Storm and Thurston [2]. The proof of this result still requires that the links in question be alternating, however. 
Our result is an extension of these results. We prove that a similar lower bound holds for a large class of knots and links, without the requirement that these knots be alternating. We do, however, need to introduce a requirement that the links be highly twisted in each twist region. Our methods use explicit deformation of a hyperbolic structure through cone manifolds, using methods of Hodgson and Kerckhoff [8; 9]. The high amount of twisting ensures that the explicit estimates in their papers and this one will apply throughout the deformation.

In order to state our results, we review some definitions concerning the diagram of a knot or link, following Lackenby [10].

Consider the diagram of a knot or link as a 4-valent graph in the plane, where associated to each vertex is over-under crossing information. A bigon region is a region of this graph bounded by only two edges.

Definition 1.1 A twist region of a diagram of a link $K$ consists of maximal collections of bigon regions arranged end to end. A single crossing adjacent to no bigons is also a twist region. Let $D_{K}$ denote the diagram of $K$. We denote the number of twist regions in a diagram by $\operatorname{tw}\left(D_{K}\right)$. See Figure 1 .

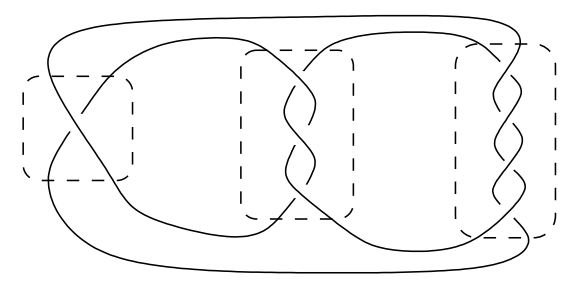

Figure 1: Twist regions of the diagram are encircled with dashed lines. For this diagram, $\operatorname{tw}\left(D_{K}\right)=3$.

Our statements concern the number of twist regions of a diagram of a knot or link. In order to rule out extraneous twist regions, we need the diagram to be reduced in the sense of the following two definitions.

First, we require the diagram to be prime. That is, any simple closed curve which meets two edges of the diagram transversely must bound a region of the diagram with no crossings.

Second, we require the diagram to be twist reduced. That is, if any simple closed curve meets the diagram transversely in four edges, with two points of intersection adjacent to one crossing and the other two adjacent to another crossing, then that simple closed 
curve must bound a region of the diagram consisting of a (possibly empty) collection of bigons arranged end to end between the crossings.

If a diagram of a hyperbolic knot or link is not prime, them some crossings are extraneous and can be removed to obtain a prime diagram. If it is not twist reduced, then a series of flypes will decrease the number of twist regions of the diagram, until we are left with a twist reduced diagram.

With these definitions, we are ready to state our results.

Theorem 1.2 There is a universal constant $C$ such that if $K$ is a knot or link admitting a prime, twist reduced diagram $D_{K}$ with at least 2 twist regions and at least $C$ crossings in each twist region, then $S^{3}-K$ is hyperbolic with volume bounded below:

$$
\operatorname{Volume}\left(S^{3}-K\right) \geq \operatorname{tw}\left(D_{K}\right)(3.3515) \text {. }
$$

Further, the universal constant $C$ is at most 113.

The upper bound on volume given by Lackenby, Agol and D Thurston is also linear in the number of twist regions of the diagram [10]. Specifically, Agol and Thurston showed

$$
\operatorname{Volume}\left(S^{3}-K\right) \leq 10 v_{3}\left(\operatorname{tw}\left(D_{K}\right)-1\right)
$$

where $v_{3}(\approx 1.01494)$ is the volume of a regular hyperbolic ideal tetrahedron.

Thus the results in this paper extend the class of knots and links for which volume is bounded above and below by linear functions of $\operatorname{tw}\left(D_{K}\right)$.

Our proof is geometric in nature. We begin with a link $L$ whose complement is geometrically explicit. We show that $S^{3}-K$ can be obtained from $S^{3}-L$ by Dehn filling. We determine a lower bound on the volume of the link complement $S^{3}-L$. We then perform hyperbolic Dehn filling by a cone deformation. Our final result is obtained by bounding the change in geometry under this cone deformation.

In Section 2, we describe the link $L$ and explain how to obtain $S^{3}-K$ from $S^{3}-L$ by Dehn filling. In Section 3, we review results on cone deformations and describe their application to our particular situation. Finally, in Section 4 we put these results together to conclude the proof of Theorem 1.2.

\section{Initial geometric estimates}

\subsection{The augmented link $L$}

Start with a prime, twist reduced diagram $D_{K}$ of a link $K$. In the rest of this paper, we will assume the diagram $D_{K}$ is fixed once and for all. To simplify notation, we will 
refer to this fixed diagram and the knot by the same symbol $K$. Thus we will write $\operatorname{tw}(K)$ to mean $\operatorname{tw}\left(D_{K}\right)$. The reader should note that the twist number $\operatorname{tw}\left(D_{K}\right)$ is dependent upon the particular choice of prime, twist reduced diagram. However, since our diagram is now fixed, this simplification should cause no confusion.

Given our diagram of $K$, we obtain a new link by adding additional link components to the diagram. At each twist region, encircle the twist region by a simple curve, called a crossing circle. See Figure 2 (a) and (b). Links with added crossing circles have been studied by many people, including Adams [1]. These links were used by Lackenby, and also by Agol and D Thurston to improve Lackenby's volume results for alternating links [10]. Provided the original diagram of $K$ was prime and twist reduced with at least two twist regions, then the link with crossing circles added is known to be hyperbolic. This can be shown either using methods of Adams [1], or directly using Andreev's theorem [13].

Let $J$ be the link with crossing circles added to each twist region of $K$. Modify the diagram of $J$ by removing pairs of crossings at each twist region, and let $L$ be this new link. Now, $S^{3}-J$ is homeomorphic, and thus isometric by Mostow-Prasad rigidity, to the manifold $S^{3}-L$. The diagram of $L$ consists of strands in the projection plane encircled in pairs by crossing circles. These strands in the projection plane only cross, if at all, in pairs at crossing circles, and here they may only cross once. We will call such a link an augmented link. See Figure 2 (c).

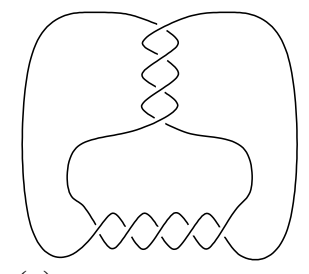

(a)

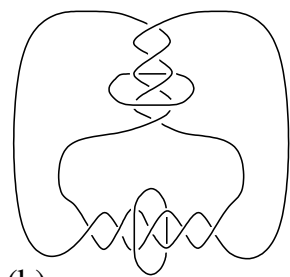

(b)

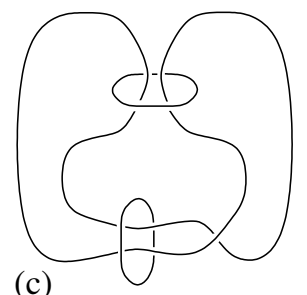

(c)

Figure 2: (a) The original link diagram $K$ (b) Crossing circles added (c) The diagram of $L$

The augmented link $L$ has a complement with nice geometric properties, allowing us to give estimates on the volume of $S^{3}-L$. Before giving these estimates, we relate $S^{3}-L$ to the complement of the original link, $S^{3}-K$. 


\subsection{Dehn filling on $S^{3}-L$}

Let $C_{i}$ be the $i$-th crossing circle of the link $L$. Take a horoball neighborhood $N_{i}$ of the cusp corresponding to $C_{i}$ in $S^{3}-L$. Then $N_{i}$ has torus boundary, with meridian $\mu$ and longitude $\lambda$. If we glue a solid torus onto $S^{3}-L-N_{i}$ in such a way that the boundary of the solid torus is glued to the boundary $\partial N_{i}$, with the curve $\mu+n_{i} \lambda$ on $\partial N_{i}$ bounding a meridional disk of the solid torus, then we obtain a new link complement in $S^{3}$. The diagram of this link is the same as that of $L$, only now $n_{i}$ full twists (ie $2 n_{i}$ crossings) have been inserted into the $i$-th twist region, and the crossing circle $C_{i}$ has been removed (see for example Rolfsen [14, Chapter 9]). Recall that the insertion of a solid torus into the manifold $S^{3}-L$ in this manner is called the Dehn filling of $S^{3}-L$ along the slope $1 / n_{i}$ on $C_{i}$.

Now, since $L$ was originally obtained from $K$ by adding crossing circles and removing crossings at twist regions in a diagram, by choosing values for the $n_{i}$ appropriately at each crossing circle, this type of Dehn filling performed at each crossing circle will give us back the original link complement $S^{3}-K$. Precisely, we perform Dehn filling of $S^{3}-L$ along the slopes $\left(1 / n_{1}, 1 / n_{2}, \ldots, 1 / n_{\mathrm{tw}(K)}\right)$ on crossing circle components to obtain the manifold $S^{3}-K$.

\subsection{Decomposition of $S^{3}-L$}

In their appendix to Lackenby's paper [10], Agol and D Thurston describe how to decompose the link complement $S^{3}-L$ into totally geodesic ideal polyhedra when $L$ happens to have no crossings of strands in the projection planes (ie in our case, when $K$ had an even number of crossings at each twist region). Their methods immediately extend to the case when single crossings of the strands in the projection plane are allowed at each twist region. In the following paragraphs, we review the main results. See also Futer and Purcell [6] for more details and pictures.

First, consider a link with no single crossings in the projection plane. Call this link $\bar{L}$. Notice that a reflection through the projection plane preserves $\bar{L}$. Thus the projection plane is a totally geodesic surface in $S^{3}-\bar{L}$ (see for example Leininger [11]). Now slice $S^{3}-\bar{L}$ along the projection plane. This breaks the manifold into two pieces with totally geodesic boundary. Each 2-punctured disc bounded by a crossing circle has been sliced in half. Next slice each of these halves and open them up into two triangles. Since triangles can be taken to be totally geodesic, the result is two identical polyhedra, $P_{1}$ and $P_{2}$, with totally geodesic faces.

The ideal polyhedra $P_{1}$ and $P_{2}$ have two kinds of faces. "White faces" come from regions of the projection plane. The triangular "shaded faces" consist of halves of the 
2-punctured discs bounded by crossing circles. These two types of faces intersect each other at right angles. The intersections are the edges of the polyhedra. The edges meet in 4-valent ideal vertices. At these vertices, two white and two shaded faces lie across from each other in pairs. See Figure 3.
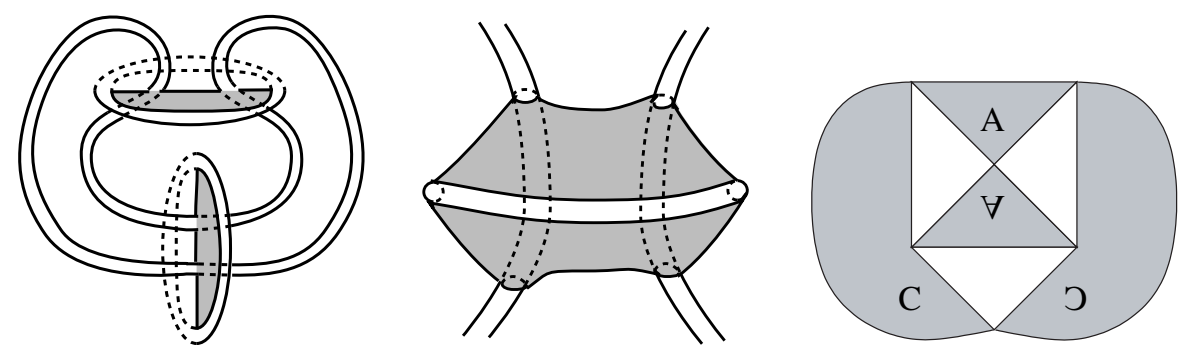

Figure 3: Decomposing $S^{3}-L$ into ideal polyhedra: first slice along the projection plane, then split the remaining halves of two-punctured disks to obtain the polyhedron on the right.

We obtain the manifold $S^{3}-\bar{L}$ from these polyhedra by reversing the slicing procedure above. First, on each $P_{i}$, form the 2 -punctured disc halves by gluing back together the two shaded triangles which were opened along their common vertex. Then glue the white faces of $P_{1}$ to the identical white faces of $P_{2}$.

By changing the gluing procedure slightly, we can obtain the manifold $S^{3}-L$ from the polyhedra $P_{1}$ and $P_{2}$ as well. Recall the link $L$ may have single crossings at some crossing circles. For each crossing circle $C_{i}$ of $L$, consider the four shaded triangles (two on $P_{1}$, and two on $P_{2}$ ) making up the disc bounded by the corresponding crossing circle of $\bar{L}$. For example, the shaded faces labelled with A in Figure 3 are two of the four triangles. The other two lie on an identical polyhedron.

If there are no single crossings at the twist region of $C_{i}$, then the gluing is the same as for $S^{3}-\bar{L}$ : Glue the triangles of $P_{i}, i=1,2$, to each other across their common vertex. For example, in Figure 3, the faces labelled A would be glued together. If there is a single crossing at $C_{i}$, glue each triangle of $P_{1}$ to the opposite one of $P_{2}$, matching vertices from the crossing circle. For example, if we replace the link on the left in Figure 3 with one with a single crossing in the top twist region, then in the gluing of the two polyhedra the top face labelled $\mathrm{A}$ in the figure on the right would be glued to the opposite face labelled A of the opposite polyhedron. This puts a "half-twist" into the manifold. See Figure 4 for another schematic picture. In either case, $S^{3}-L$ is then obtained by gluing corresponding white faces of $P_{1}$ and $P_{2}$ together, as in the gluing of $S^{3}-\bar{L}$.

Algebraic ${ }^{3} \mathcal{G}$ Geometric Topology, Volume 7 (2007) 

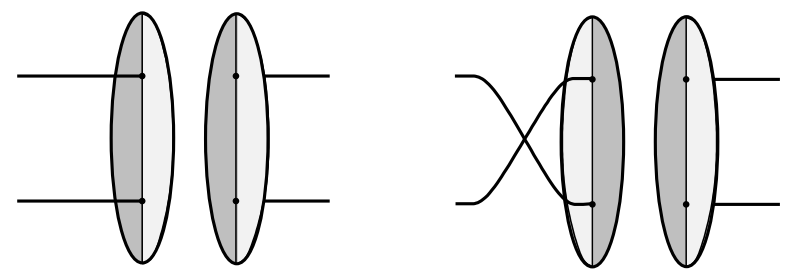

Figure 4: Left: Gluing 2-punctured discs with no crossings Right: Gluing 2-punctured discs with a single crossing

Now, we wish to find lower bounds on volume. We will give a rough lower bound first by considering the cusp volume of $S^{3}-L$.

\subsection{Volume estimate for $S^{3}-L$}

To give an estimate on the cusp volume of $S^{3}-L$, we must analyze neighborhoods of the ideal vertices of the polyhedra. These neighborhoods were analyzed quite carefully with Futer in [6]. Again we record the results here.

A horospherical torus about a cusp of the manifold $S^{3}-L$ will intersect the polyhedra $P_{1}$ and $P_{2}$ in rectangles. This is because at a cusp, ideal vertices of $P_{1}$ and $P_{2}$ meet. These vertices are all $4-$ valent, meaning their intersection with a horosphere is a quadrilateral, and all faces of the $P_{i}$ meet at right angles, forcing the quadrilateral to be a rectangle. The sides of this rectangle can be colored according to the color of the faces of intersection. Thus each rectangle will have two "white sides" across from each other, and two "shaded sides" across from each other. When we glue the polyhedra together to form $S^{3}-L$, we glue these rectangles along white and shaded sides. Thus each cusp of $S^{3}-L$ is tiled by rectangles. The number and size of these rectangles will give us a volume estimate.

The following two lemmas are essentially Lemmas 2.3 and 2.6 of [6].

Lemma 2.1 A horospherical torus about a cusp in $S^{3}-L$ corresponding to a crossing circle is tiled by two rectangles. These rectangles are given by the intersection of the horospherical torus with $P_{1}$ and $P_{2}$.

- A longitude of the crossing circle is isotopic to the curve given by stepping along two shaded sides of the rectangles.

- A meridian is isotopic to the curve given by a white side of a rectangle plus $\epsilon$ times a shaded side, where $\epsilon=0, \pm 1$ depending on whether the crossing circle bounded no crossings, or one crossing in the positive or negative direction, respectively. 
Lemma 2.2 A horospherical torus about a cusp in $S^{3}-L$ corresponding to a link component $L_{i}$ in the projection plane is tiled by $2 C\left(L_{i}\right)$ rectangles, where $C\left(L_{i}\right)$ is the number of crossing circles, counted with multiplicity, through which $L_{i}$ passes in the diagram of $L$.

When $K$ is a knot with diagram $D_{K}$, there is just one link component in the projection plane, and so it passes through each crossing circle twice. Since the number of crossing circles in the diagram of $L$ is $\operatorname{tw}(K)$, in this case the number of rectangles in the cusp of $S^{3}-L$ corresponding to the link component in the projection plane is $4 \mathrm{tw}(K)$.

When $K$ is a link, we still have two strands passing through each crossing circle. Thus the total number of rectangles in all the cusps of $S^{3}-L$ corresponding to link components on the projection plane is still $4 \mathrm{tw}(K)$.

We record these results in the following lemma.

Lemma 2.3 There are a total of $4 \mathrm{tw}(K)$ rectangles tiling all horospherical tori about the cusps of $S^{3}-L$ corresponding to link components in the projection plane.

We need the sizes of these rectangles. Again these results were previously computed. The following is essentially Corollary 3.9 of [6].

Lemma 2.4 There exists a choice of horospheres expanded about all cusps of $S^{3}-L$ so that given this expansion, the length of any shaded side of a rectangle is exactly one. The length of any white side of a rectangle is at least one.

These lemmas immediately give the following proposition.

Proposition 2.5 Let $K$ be a link with a prime, $t$ wist reduced diagram with $\operatorname{tw}(K) \geq 2$ twist regions. Then the augmented link $L$ obtained by adding a crossing circle to the diagram of $K$ at each twist region has complement $S^{3}-L$ with cusp volume at least $3 \mathrm{tw}(K)$.

Proof We can compute the cusp volume by computing the volume of a region in the universal cover $\mathbb{M}^{3}$ of $S^{3}-L$ which projects to the cusp in a one-to-one manner.

Consider the upper half space model of $\mathbb{H}^{3}$. For any cusp, we can conjugate so that the point at infinity in this model of $\mathbb{M}^{3}$ projects to that cusp under the covering map. Then the rectangles tiling that cusp lift to give rectangles tiling a horosphere about infinity. For each rectangle on the cusp, choose a representative on the horosphere projecting 
to that rectangle. Then the volume of the cusp is given by the sum of the volumes of regions of $\mathbb{M}^{3}$ lying over these rectangles.

By Lemma 2.4, the volume over any of these rectangles is at least as large as the volume lying over the square with side length 1 . A calculation shows that the volume lying over a square of side length 1 is $1 / 2$. So the volume is at least $1 / 2$ times the total number of rectangles tiling all cusps of $S^{3}-L$.

To determine the total number of rectangles tiling all cusps of $S^{3}-L$, we use the previous lemmas. By Lemma 2.3, there are $4 \mathrm{tw}(K)$ rectangles tiling all cusps coming from link components in the projection plane. By Lemma 2.1, there are 2 rectangles per crossing circle. But there are $\operatorname{tw}(K)$ total crossing circles in the diagram of $L$. Hence there are $2 \operatorname{tw}(K)$ rectangles tiling cusps coming from crossing circles. So the total number of rectangles tiling all cusps of $S^{3}-L$ is $6 \mathrm{tw}(K)$.

Thus the total cusp volume is at least $6 \operatorname{tw}(K)(1 / 2)=3 \operatorname{tw}(K)$.

We obtain a better estimate on volume by combining this cusp estimate with Böröczky's lower bound for the density of a horoball packing in hyperbolic space [3]. By his result, the volume of a maximal cusp neighborhood in a hyperbolic 3-manifold $M$ is at most $\sqrt{3} /\left(2 v_{0}\right) \approx 0.853276$ times the volume of the manifold $M$, where $v_{0}$ is the volume of a regular ideal hyperbolic tetrahedron. Thus the volume of $S^{3}-L$ is at least $(3 \mathrm{tw}(K)) /(0.853276) \approx 3.51586 \mathrm{tw}(K)$.

Corollary 2.6 Let $K$ be a link with a prime, twist reduced diagram with $\operatorname{tw}(K) \geq 2$ twist regions. Then the augmented link $L$ obtained by adding a crossing circle to the diagram of $K$ at each twist region has complement $S^{3}-L$ with volume at least (3.51586) $\operatorname{tw}(K)$.

\section{Deformation through cone manifolds}

\subsection{Hyperbolic Dehn filling}

In the previous section, we obtained a hyperbolic link whose geometry could be determined explicitly. This hyperbolic link was related to our original link by a Dehn filling, ie by gluing solid tori into the cusps corresponding to crossing circles.

Thurston showed that most Dehn fillings on components of a hyperbolic link complement can be obtained by a hyperbolic Dehn filling, that is, by deforming the complete hyperbolic geometric structure on the cusped manifold through incomplete hyperbolic structures, yielding a new manifold with a final complete hyperbolic structure [15]. 
Hodgson and Kerckhoff were able to make this explicit in [9]. They showed that if the normalized lengths of the slopes along which the hyperbolic Dehn filling is performed are each longer than a universal constant, then hyperbolic Dehn filling is possible. In particular, in this case of long slopes, a special type of deformation through incomplete hyperbolic structures exists, called a hyperbolic cone deformation. In a hyperbolic cone deformation, each of the intermediate incomplete structures of the deformation is a hyperbolic cone manifold.

For a complete description of hyperbolic cone manifolds and the metrics involved, see Hodgson and Kerckhoff [8]. For our purposes, a hyperbolic cone manifold $M$ admits a smooth hyperbolic metric everywhere except along a link $\Sigma$. At each link component $\Sigma_{i}$ of $\Sigma$, a tubular neighborhood has meridional cross section which is a 2-dimensional hyperbolic cone, with cone angle $\alpha_{i}$. The cone manifold structure on $M$ is locally parameterized by the collection of angles $\left(\alpha_{1}, \alpha_{2}, \ldots, \alpha_{N}\right)$.

In our case, recall that we wish to perform Dehn filling on $S^{3}-L$ to obtain $S^{3}-K$. The fillings are performed along the slopes $1 / n_{i}$ on the crossing circles. Thus our singular locus $\Sigma$ will consist of the cores of the crossing circles. Provided our slopes are long enough to meet the requirements found in [9], we will obtain a cone deformation of the hyperbolic structure on $S^{3}-L$ with initial cone angles all 0 . Each cone angle will increase strictly monotonically to cone angle $2 \pi$ under the deformation.

In [9], Hodgson and Kerckhoff found bounds on the change of volume under certain cone deformations, in particular those with a single component of the singular locus $\Sigma$. Since we are filling along multiple cusps, with multiple components of $\Sigma$ (one per crossing circle), we will need versions of the results in [9] which allow filling along more than one cusp. We state those results here.

\subsection{Change of volume}

The following theorem is Theorem 6.5 of [9], modified for the case of multiple components of the singular locus $\Sigma$. The notation is from that paper. In particular,

$$
H(z)=\frac{1+z^{2}}{3.3957 z\left(1-z^{2}\right)},
$$

and

$$
\widetilde{G}(z)=\frac{\left(1+z^{2}\right)^{2}}{6.7914 z^{3}\left(3-z^{2}\right)}
$$

Algebraic ${ }^{3} \mathcal{G}$ Geometric Topology, Volume 7 (2007) 
Theorem 3.1 Let $X$ be a cusped hyperbolic 3-manifold and $M$ a hyperbolic 3manifold which can be joined by a smooth family of hyperbolic cone manifolds with cone angles $0 \leq \alpha_{i} \leq 2 \pi$ along the $i$-th component $\Sigma_{i}$ of a link $\Sigma$. Suppose that $\alpha_{i} \ell_{i} \leq 0.5098$ holds throughout the deformation, where $\ell_{i}$ denotes the length of $\Sigma_{i}$. Then the difference in volume

$$
\Delta V=\operatorname{Volume}(X)-\operatorname{Volume}(M)
$$

satisfies

$$
\Delta V \leq \sum_{i} \int_{\widehat{z}_{i}}^{1} \frac{H^{\prime}(w)}{8 H(w)(H(w)-\widetilde{G}(w))} d w .
$$

Here $\widehat{z}_{i}=\tanh \left(\hat{\rho}_{i}\right), \hat{\rho}_{i}$ is the unique solution of $1.69785 \tanh \left(\hat{\rho}_{i}\right) / \cosh \left(2 \widehat{\rho}_{i}\right)=2 \pi \hat{\ell}_{i}$ with $\hat{\rho}_{i} \geq 0.531$, and $\hat{\ell}_{i}$ is the length of $\Sigma_{i}$ in $M$.

Since this version of the theorem is somewhat different from that in [9], we outline its proof in the appendix, Section 5.

\section{The volume estimate}

In this section, we will find conditions under which Theorem 3.1 will apply. Using these conditions, we can complete the proof of Theorem 1.2.

For Theorem 3.1 to apply, we first need a smooth cone deformation from the manifold $S^{3}-L$ to the manifold $S^{3}-K$. We also need each pair of cone angle $\alpha_{i}$ and length of singular locus component $\ell_{i}$ to satisfy the inequality $\alpha_{i} \ell_{i} \leq 0.5098$.

In fact, the existence of the deformation and the bound on $\alpha_{i} \ell_{i}$ are both given by Hodgson and Kerckhoff in Theorem 5.12 of [9]. (More accurately, these are given in the proof of that theorem.) In our situation, this theorem states that provided the slopes $1 / n_{i}$ along which we perform Dehn filling have normalized length at least $\sqrt{2(56.4696)} \approx 10.628$, then a cone deformation exists with initial point the complete hyperbolic structure on $S^{3}-L$, and extends to give the complete hyperbolic structure on $S^{3}-K$ with each cone angle equal to $2 \pi$.

Normalized length is defined as follows.

Definition 4.1 Let $s$ be a slope on a cusp with cusp torus $T$. Let $\alpha$ be a geodesic representative of $s$ on $T$. The normalized length of $s$ is defined to be the length of $\alpha$ divided by the square root of the area of $T$ :

$$
\text { Normalized length }(s)=\frac{\text { Length }(\alpha)}{\sqrt{\operatorname{Area}(T)}} .
$$


At the $i$-th crossing circle, recall we perform Dehn filling along the curve $\sigma_{i}=\mu+n_{i} \lambda$, where $\mu$ is a meridian of a torus about this crossing circle, and $\lambda$ is a longitude. Thus we need to estimate the normalized length of this curve, to ensure it is at least $\sqrt{2(56.4696)}$.

In Section 2, Lemma 2.1, we saw that a torus about a crossing circle is tiled by two rectangles with white and shaded sides, and meridian and longitude given by steps along those sides. The longitude $\lambda$ is given by two steps along shaded sides. If the crossing circle bounds a single crossing, then $\mu$ is given by one step along a white side, plus or minus a step along a shaded side, and $\sigma_{i}$ is given by a step along a white side plus (or minus) $2 n_{i}+1$ steps along shaded sides. If the crossing circle bounds no single crossing, then $\mu$ is given by one step along a white side, and $\sigma_{i}$ is given by a step along a white side plus (or minus) $2 n_{i}$ steps along shaded sides. In either case, note that if $c_{i}$ is the number of crossings in the $i$-th twist region, then the curve $\sigma_{i}$ is given by one step along a white side plus (or minus) $c_{i}$ steps along shaded sides.

Let $w$ denote the length of a white side and let $s$ denote the length of a shaded side. Since the area of the torus is $2 s w$, the normalized length $\widehat{L}_{i}$ of $\sigma_{i}$ is given by

$$
\widehat{L}_{i}=\sqrt{\frac{w}{2 s}+\frac{c_{i}^{2} s}{2 w}} .
$$

This will be a minimum when $w / 2 s$ equals $c_{i} / 2$, and that minimum value is $\sqrt{c_{i}}$. Hence we need $c_{i}$ large enough that

$$
\sqrt{c_{i}} \geq \sqrt{2(56.4696)}=\sqrt{112.9392} .
$$

Thus $c_{i} \geq 113$ will be sufficient.

Then Theorem 5.12 of [9] will apply to $X=S^{3}-L$ to give the desired cone deformation and bounds on the $\alpha_{i} \ell_{i}$. Hence Theorem 3.1 applies to $X=S^{3}-L$ and $M=S^{3}-K$, and we find

$$
\operatorname{Volume}(X)-\operatorname{Volume}(M) \leq \sum_{i=1}^{\operatorname{tw}(K)} \int_{\widehat{z}_{i}}^{1} \frac{H^{\prime}(w) d w}{8 H(w)(H(w)-\widetilde{G}(w))}
$$

Note the sum is over all components of the singular locus. In our case, these correspond to the crossing circles, and there are exactly $\operatorname{tw}(K)$ of these.

In the $i$-th cusp, the value of $z$ decreases from 1 to the value $\widehat{z}_{i}$. Recall from the statement of Theorem 3.1 that $\widehat{z}_{i}=\tanh \left(\hat{\rho}_{i}\right)$, where $\hat{\rho}_{i}$ is the unique solution of some equation such that $\hat{\rho}_{i} \geq 0.531$. In particular, $\hat{\rho}_{i}$ is guaranteed to be at least 0.531 . So 
$\widehat{z}_{i}$ is at least $z_{1}=\tanh (0.531)$. Thus the decrease in volume is at most:

$$
\sum_{i=1}^{\operatorname{tw}(K)} \int_{z_{1}}^{1} \frac{H^{\prime}(w) d w}{8 H(w)(H(w)-\widetilde{G}(w))} \leq \sum_{i=1}^{\operatorname{tw}(K)} 0.16436=\operatorname{tw}(K)(0.16436)
$$

Hence $\quad \operatorname{Volume}(M) \geq \operatorname{Volume}(\mathrm{X})-\operatorname{tw}(K)(0.16436)$.

Also, we know by Corollary 2.6 that $\operatorname{Volume}(X) \geq(3.51586) \operatorname{tw}(K)$. Hence we obtain our final result:

$$
\operatorname{Volume}(M) \geq(3.51586) \operatorname{tw}(K)-\operatorname{tw}(K)(0.16436)=\operatorname{tw}(K)(3.3515)
$$

This concludes the proof of Theorem 1.2 which we restate.

Theorem 4.2 Let $K$ be a link in $S^{3}$ admitting a prime, twist reduced diagram with $\operatorname{tw}(K) \geq 2$ twist regions and at least 113 crossings per twist region. Then the volume of $S^{3}-K$ satisfies:

$$
\operatorname{Volume}\left(S^{3}-K\right) \geq \operatorname{tw}(K)(3.3515)
$$

\section{Appendix}

In this appendix, we sketch the proof of Theorem 3.1.

The proof is identical to that in [9], except for small modifications that need to be made for the case in which the cone manifold has multiple components of the singular locus.

In particular, the following changes to that proof need to be made. First, in [9], the cone deformation was parameterized by $t=\alpha^{2}$. When there are multiple components, we can no longer guarantee that the deformation is parameterized by $t=\alpha_{j}^{2}$ for each $\alpha_{j}$. We do know that some parameterization exists for which the calculations of [9] go through (see the comment at the bottom of page 41 in [9] and Purcell [12]), but we don't know specifically what that parameterization will be. Thus we need to modify the calculations to be independent of parameterization.

We also need to make modifications to calculations which used the area estimate for a single cusp, Theorem 4.4 of [9]. As stated in that theorem, the lower bound for the area is half as large when the singular locus has multiple components. Thus we replace Corollary 5.1 of [9] with half that estimate:

$$
\alpha_{i} \ell_{i} \geq \frac{1}{2}\left(3.3957 \frac{\tanh (R)}{\cosh (2 R)}\right)=1.6978 \frac{\tanh (R)}{\cosh (2 R)} .
$$


We recompute calculations using this new estimate.

With these two modifications, the proof goes through nearly as written in [9]. Following their notation, we begin by letting $u_{i}=\alpha_{i} / \ell_{i}$. Hodgson and Kerckhoff bounded the change in $u_{i}$ by finding bounds on $d u_{i} / d t$, in Proposition 5.6. Rather than take derivatives with respect to time $t$, we take derivatives with respect to cone angle $\alpha_{i}$ in the $i$-th cusp. This is independent of parameterization. The half of Proposition 5.6 of [9] necessary for Theorem 3.1 becomes:

$$
\frac{1}{\alpha_{i}} \frac{d u_{i}}{d \alpha_{i}} \leq 4 \widetilde{G}\left(z_{i}\right)
$$

where the function $\widetilde{G}(z)$ is defined as in (2).

Now we are ready to modify the proof of Theorem 6.5 of [9] directly. From the Schläfli formula (see Cooper, Hodgson and Kerckhoff [5] and Hodgson [7]), the change in volume $V$ of a hyperbolic cone manifold during a deformation satisfies

$$
d V=-\frac{1}{2} \sum_{i} \ell_{i} d \alpha_{i}=-\sum_{i} \frac{\alpha_{i} d \alpha_{i}}{2 u_{i}}
$$

We will rewrite $u_{i}$ in terms of the function $H$, where $H$ is given by (1). $H$ was defined so that $H(\tanh (R))$ is the reciprocal of the function

$$
h(R)=3.3957 \frac{\tanh (R)}{\cosh (2 R)},
$$

which appears in the inequality (3). Provided $R \geq 0.531$, this function $h(R)$ is strictly decreasing, and thus we may define its inverse for $x$ in the interval $(0, h(0.531)] \approx$ $(0,1.019675]$. Since $\alpha_{i} \ell_{i} \leq 0.5098,2 \alpha_{i} \ell_{i} \leq h(0.531)$. We define $\rho_{i}=h^{-1}\left(2 \alpha_{i} \ell_{i}\right)$. Letting $z_{i}=\tanh \left(\rho_{i}\right)$, we have $u_{i}=2 \alpha_{i}^{2} H\left(z_{i}\right)$. So the term $-\alpha_{i} /\left(2 u_{i}\right)$ equals $-1 /\left(4 \alpha_{i} H\left(z_{i}\right)\right)$, and we obtain:

$$
d V=-\sum_{i} \frac{1}{4 \alpha_{i} H\left(z_{i}\right)} d \alpha_{i}
$$

Now, by inequality (4), we have

$$
\frac{1}{\alpha_{i}} \frac{d u_{i}}{d \alpha_{i}} \leq 4 \widetilde{G}\left(z_{i}\right)
$$

Thus

$$
\frac{1}{\alpha_{i}} \frac{d u_{i}}{d \alpha_{i}}=\frac{1}{\alpha_{i}} \frac{d}{d \alpha_{i}}\left(2 \alpha_{i}^{2} H\left(z_{i}\right)\right)=4 H\left(z_{i}\right)+2 \alpha_{i} H^{\prime}\left(z_{i}\right) \frac{d z_{i}}{d \alpha_{i}}
$$

Algebraic 83 Geometric Topology, Volume 7 (2007) 
Hence

$$
\alpha_{i} H^{\prime}\left(z_{i}\right) \frac{d z_{i}}{d \alpha_{i}} \leq 2\left(\widetilde{G}\left(z_{i}\right)-H\left(z_{i}\right)\right)
$$

which implies

$$
-\frac{1}{\alpha_{i}} \geq \frac{H^{\prime}\left(z_{i}\right)}{2\left(H\left(z_{i}\right)-\widetilde{G}\left(z_{i}\right)\right)} \frac{d z_{i}}{d \alpha_{i}} .
$$

To see that the direction of the inequalities is correct, notice that $H\left(z_{i}\right)-\widetilde{G}\left(z_{i}\right)$ is positive.

Now, multiply the inequality (6) by $1 /\left(4 H\left(z_{i}\right)\right)$. This bounds each term in equation (5).

$$
d V \geq \sum_{i} \frac{H^{\prime}\left(z_{i}\right)}{8 H\left(z_{i}\right)\left(H\left(z_{i}\right)-\widetilde{G}\left(z_{i}\right)\right)} d z_{i} .
$$

We integrate over the deformation. As $\alpha_{i}$ increases from 0 to $2 \pi, z_{i}$ decreases from 1 to $\widehat{z}_{i}$. When $\alpha_{i}$ has reached $2 \pi$, it remains $2 \pi$ and there is no further contribution.

Hence

$$
\Delta V \leq \sum_{i} \int_{\widehat{z}_{i}}^{1} \frac{H^{\prime}\left(z_{i}\right)}{8 H\left(z_{i}\right)\left(H\left(z_{i}\right)-\widetilde{G}\left(z_{i}\right)\right)} d z_{i} .
$$

\section{References}

[1] C C Adams, Augmented alternating link complements are hyperbolic, from: "Lowdimensional topology and Kleinian groups (Coventry/Durham, 1984)", London Math. Soc. Lecture Note Ser. 112, Cambridge Univ. Press, Cambridge (1986) 115-130 MR903861

[2] I Agol, N Dunfield, P Storm, W P Thurston, Lower bounds on volumes of hyperbolic Haken 3-manifolds arXiv:math.DG/0506338

[3] K Böröczky, Packing of spheres in spaces of constant curvature, Acta Math. Acad. Sci. Hungar. 32 (1978) 243-261 MR512399

[4] C Cao, G R Meyerhoff, The orientable cusped hyperbolic 3-manifolds of minimum volume, Invent. Math. 146 (2001) 451-478 MR1869847

[5] D Cooper, CD Hodgson, SP Kerckhoff, Three-dimensional orbifolds and cone-manifolds, MSJ Memoirs 5, Mathematical Society of Japan, Tokyo (2000) MR1778789With a postface by Sadayoshi Kojima

[6] D Futer, J S Purcell, Links with no exceptional surgeries arXiv:math.GT/0412307

[7] C D Hodgson, Degeneration and regeneration of geometric structures on 3-manifolds, $\mathrm{PhD}$ thesis, Princeton Univ. (1986)

[8] C D Hodgson, S P Kerckhoff, Rigidity of hyperbolic cone-manifolds and hyperbolic Dehn surgery, J. Differential Geom. 48 (1998) 1-59 MR1622600 
[9] C D Hodgson, S P Kerckhoff, Universal bounds for hyperbolic Dehn surgery, Ann. of Math. (2) 162 (2005) 367-421 MR2178964

[10] M Lackenby, The volume of hyperbolic alternating link complements, Proc. London Math. Soc. (3) 88 (2004) 204-224 MR2018964With an appendix by Ian Agol and Dylan Thurston

[11] C J Leininger, Small curvature surfaces in hyperbolic 3-manifolds, J. Knot Theory Ramifications 15 (2006) 379-411 MR2217503

[12] J S Purcell, Cusp shapes under cone deformation arXiv:math.GT/0410233

[13] JS Purcell, Cusp Shapes of Hyperbolic Link Complements and Dehn Filling, PhD thesis, Stanford University (2004)

[14] D Rolfsen, Knots and links, Mathematics Lecture Series 7, Publish or Perish, Berkeley, CA (1976) MR0515288

[15] W P Thurston, The Geometry and Topology of Three-Manifolds, Princeton Univ. Math. Dept. Notes (1979)

[16] J R Weeks, Snappea http: //www .geometrygames.org/SnapPea/

Department of Mathematics, 1 University Station C1200, University of Texas at Austin Austin, TX 78712

jpurcell@math. utexas.edu

Received: 21 April 2006 Revised: 3 January 2007 